\title{
Relationship of Knowledge on Healthy Lifestyle to Dietary Practices and Physical Activity as Moderated by Age
}

\author{
Mary Anne Grace M. Dorado ${ }^{1}$, Abraham P. Racca ${ }^{2}$ \\ ${ }^{1,2}$ Adventist University of the Philippines \\ magmdorado@aup.edu.ph
}

\begin{abstract}
Lifestyle - related diseases remained to be on the top ten killer according to the World Health Organization. Literature indicates that diseases are highly related to lifestyle practices. On the other hand, optimal health is a perceived goal of the greater population, however, putting it into practice is a challenge. The study determined the following: 1) the level of knowledge on healthy lifestyle, dietary practices and physical activity; 2) the relationship of knowledge on healthy lifestyle to dietary practices and physical activities, and 3) the relationship of knowledge to dietary practice and relationship of knowledge to physical activities according to age group. A Descriptive-correlational design was employed. The data were gathered among 66 randomly selected university students staying in the dormitories; who are at least 18 years of age, in which 24 are males and 42 are females, and 40 have earned at most a bachelor's degree and 26 have at least earned units in a master's program. Utilizing adapted instruments, respondents were scored on a 12-item instrument in knowledge where in each correct answer correspond to one point, 27-item instrument on healthy lifestyle in terms of diet where in each appropriate response correspond to one point, and 4-item instrument on healthy lifestyle in terms of physical activities. In turn, the highest possible score of knowledge, diet, and physical activities are 12, 27, and 4, respectively. Descriptive results revealed that respondents have high knowledge on healthy lifestyle, have fair dietary practices and moderate practice of physical activities. Furthermore, knowledge on healthy lifestyle is not significantly correlated to dietary practices physical activities. However, a significant relationship was found when respondents were grouped according to age; only those 24 years old and above were found to have significant results. Hence, a recommendation to increase the accessibility and reinforcement values of exercise and physical activity that will have immediate and long term health benefits for college students.
\end{abstract}

Keywords: Knowledge, Dietary Practices, Physical Activity.

\section{INTRODUCTION}

According to World Health Organization in 2018, ages 30-69 years old are the population mostly affected by non-communicable diseases; hence, attention is given on this age group in 
health promotions. However, despite the efforts on the prevention of non-communicable diseases, no studies are focused yet on young adults such as these university students (Lupi, S., et al., 2015). More often than not, behaviors picked up during this stage persist until adult life.

Moreover, students staying in the university may have to play a balancing act between keeping their good health and being on top of their academic classes. This scenario, more often, puts them in a situation wherein their health takes the back seat. The university becomes their new home, a place where they prepare for their future roles within the communities and the society as a whole, thereby passing the responsibility of their parents to the university in providing the opportunity to explore new experiences and improve their health. ASCD, Centers for Disease Control and Prevention (CDC) in 2014, highlighted the challenge that was given to the education sector to ensure that all students are supported, healthy and safe. Opportunities for translation of their knowledge into practice are therefore provided. The practice of healthy lifestyle which includes the engagement in any form of physical activity is a known factor to good growth and a better development of mental ability of the students. However, a research study showed that there is no significant relationship between the knowledge and its practice and showed some barriers such as time constraints and concerns on time management among others (Zakaria and Abidin, 2014).

Recent research studies showed how health is being practiced by university students. The result showed skipping breakfast were often associated with physical inactivity in men and low personal control; and knowledge of exercise-heart link in women are associated with physical inactivity (Pengpid et al., 2015). Another study on physical activity which was being influenced by self-discipline, social support and the accessibility and availability of opportunities (Simons et al., 2014). These factors may have been the same factors that has contributed to poor healthy lifestyle practices despite the high level of knowledge that the university students acquired. Previous studies on the cause of physical inactivity may have been described for the general population of the university students. Moreover, only a few studies have examined the relationship of knowledge of healthy lifestyle to practice being utilized by a specific group of university students. Therefore, this study is to assess whether the knowledge on healthy lifestyle has a relationship on dietary practices and physical activity among university students and assess whether age could make a difference.

The study assessed whether a) the knowledge on healthy lifestyle has a relationship on the dietary practices and physical activity of the college students and b) the knowledge on 

number

healthy lifestyle, dietary practices and physical activity is moderated by age. The study findings may provide evidence to strengthen and revisit the health policies of the university.

\section{LITERATURE REVIEW}

According to the World Health Organization in 2018, noncommunicable diseases are the cause of mortality among 41 million people each year, which is $71 \%$ of all deaths globally, such as cancers, respiratory diseases and diabetes, respectively, of which is caused by physical inactivity, and unhealthy diets together with other factors. Globally, this poses a burden on human population as it has an impact on financial cost of the family, mortality and morbidity factors, thus affecting the longevity of the individual.

The department of health also has different health programs to raise awareness of noncommunicable diseases and how to combat these, however, because of different factors, these health problems still continue to rise. There must be a problem somewhere in putting the knowledge into practice. A study was conducted among Ischemic heart disease patients and majority of the patients verbalized their awareness of the need for lifestyle change, however, failed to translate them into practice because of some misconceptions (Al-Tamimi, Ba-Omar, \& Nadar, 2017).

Studies shows that university students, especially those who live alone in their dormitories away from home are those who have difficulty in the practice of healthy dietary behaviors. In the Philippines, a research study conducted by the Department of Science and Technology, Food and Nutrition Research Institute (DOST-FNRI) in 2013, showed that almost half of the Filipino adults are eating meals away from home and the majority of them who practiced this are among students, 19 to 29 years old females, which resulted to overweight and obesity with high waist circumference and elevated triglycerides. They are most likely to spend less time in physical activities (Bagordo, Grassi, Serio, Idolo, \& De Donno, 2013) due to attention to weights, the stress brought about by academic area and the beliefs (Driskell, Kim, Goebel, 2005) . This scenario may be different for students who live at home with their parents and consumed home cooked meals, fruits, vegetables, and meat.

The practice of healthy lifestyle which includes the engagement in any form of physical activity is a known factor to good growth and a better development of mental ability of the students. However, according to Zakaria and Abidin (2014), knowledge and its practice has no significant relationship; time constraints and concerns on time management were noted as barriers among others. 
However, a recent study showed that health responsibility and control of stress had a better link with physical activity and proper nutrition which means, when an older adult college student have a higher health responsibility, the more evident is the practice of a healthy lifestyle (Al-Khawaldeh, 2014). This factor may provide a clear message that age could be a link to a better practice of healthy lifestyle, thus the need for further research study.

Further, despite the fact that ages 30-69 years old are the most affected by non-communicable diseases, and the efforts on the prevention of non-communicable diseases, no studies focused on young adults such as the university students.

\section{METHODS}

This quantitative study employed the descriptive-correlation design intended to examine the relationship of knowledge on healthy lifestyle to the dietary practices and physical activity of the university students staying in the dormitories, living away from their homes. This design was utilized to determine how a set of variables is related or to test hypothesis regarding expected relations. This is definitely an excellent way for finalizing results and proving or disproving a hypothesis, because it is about asking people for their opinions in a structured way using the pen and paper instruments. Furthermore, it involves collecting and describing data to determine whether and to what degree a relationship exists between two or more variables.

The data were gathered among 66 randomly selected respondents who are at least 18 years of age, in which 24 are males and 42 are females, and 40 have earned at most a bachelor's degree and 26 have at least earned units in a master's program. All of the respondents are staying in the dormitories. The sampling techniques employed by the researcher in this study were simple random by drawing lots that shows an unbiased representation of groups and convenient sampling that is close to hand and proximity to the researcher, to increase the generalizability of the results. The researcher was able to retrieve 66 out of 78 distributed questionnaires which yielded to $85 \%$ retrieval.

At the onset of the gathering of data, the researcher presented the study to the Ethical review board of the university and was released a letter to conduct the study. The researcher sent the endorsement letters to the chosen dormitories and met some of the students personally to administer the questionnaires. As soon as the researcher retrieved the answered questionnaires, the data was encoded, sent to the statistician to be analyzed, and summarized; results were then given back to the researcher for interpretation. 

number

The research instrument utilized in this study was an adapted instrument. Minor revisions were made on the instrument to suit to the type of respondents. Revisions generated were based on the dimensions of the variables. The instrument utilized in this study first went through a validation by the panel of experts before the pilot study was conducted. The validation of instrument was a process to see whether the items were clear and unequivocal, adequate to collect data, not biased and relevant to the study. The researcher personally administered and gathered some of the instruments while others were distributed and gathered by research assistants with actual experience and relevant qualifications.

The instruments of the study was comprised of the following sections: Part I of the questionnaire dealt with the respondents' demographic profile questionnaire (DPQ). This instrument consisted of three items: age, sex, and educational achievement. Part II contained the knowledge on healthy lifestyle. The indicators included were on the knowledge on diet specifically on fruits and vegetables, sugar, fat, staple food, animal products, dairy products, beans and bean products, physical activity, and sports.

Part III contained the diet practice and physical activity. Dietary practices were assessed using the following indicators: Eating habits, consumption of fruits and vegetables, fat, sugar, salt, drinks, and water. The indicators for physical activity were: vigorous exercise for 20 minutes, less vigorous for 30 minutes, walking for 30 minutes, and vigorous activity (manual work). These are the activities that were assessed as the form of physical activities the subjects are engaged in. These were gauged from no activity to hard physical activity that makes them breathe much harder than normal.

The respondents were scored on a; 12-item instrument in knowledge where in each correct answer correspond to one point, 27-item instrument on healthy lifestyle in terms of diet where in each appropriate response correspond to one point and a 4 - item instrument on healthy lifestyle in terms of physical activities. The moderating variables that were considered were the age, sex, and educational attainment.

To present and summarize the data in accordance with the specific sub-problems set in the study through the aid of Statistical Package for Social Science (SPSS), the following techniques or tools were used to treat the data statistically.

Descriptive statistics including frequencies, percentages, means, and standard deviations were used. T-test and ANOVA were employed to verify the significant differences in dietary practice and physical activity when classified according to age, sex, and educational attainment. 
A correlation test via Pearson Product-moment correlation coefficient (r) was used to explain the relationship between knowledge level and Healthy lifestyle (in terms of diet) level, and knowledge level and physical activity level

\section{RESULTS}

The Level of Knowledge on Healthy Lifestyle, Dietary Practices and Physical Activity.

Table 1 presents the level of knowledge on healthy lifestyle and descriptive results revealed that respondents were highly knowledgeable on healthy lifestyle. The study showed that the subjects who responded on the following questions: physical activities are good for one's health which is $100 \%$, followed by choosing a diet with a lot of fresh fruit and vegetables is good for one's health (97\%) and eating a lot of sugar is not good for one's health $(95.5 \%)$. These are in contrast to their lowest scores such as eating a variety of foods is good for one's health, consuming milk and dairy products is good for one's health and choosing a diet with a lot of staple foods with scores of $68.2,66.7$, and 54.5 respectively.

Moreover, the study showed top three areas that may have determined the level of dietary practices of the respondents, namely; adding sugar to their breakfast meals (80.3\%), skipping lunch more than once a week (77.3\%) and regularly eating pre-prepared meals such as canned food, choosing low - fat products when available (68.2\%). The respondents who are staying in the dormitories used to dine in the university cafeteria, however their food intake outside the cafeteria may not be monitored. Skipping breakfast may have been the biggest barrier toward healthy eating, the result of the study conducted among medical students (Bano, AlShammari E., Fatima \& AlShammari, NA, 2013). Previous studies showed those who skipped breakfast tends to consume unhealthy foods resulting to weight gains (Utter, Scragg, Mhurchu \& Schaaf, 2007).

Furthermore, the study showed that respondents engaged in briskwalking for 30 minutes three times a week. The result may have been possible because the dormitories are situated far from the classrooms and other areas of possible destination of the students.

Table 1. Respondent's level of Knowledge, Diet Practices, and Physical Activity

\begin{tabular}{rccc}
\hline Variable & $N$ & Mean & $S D$ \\
\hline Knowledge & 66 & 9.97 & 1.37
\end{tabular}


Abstract Proceedings International Scholars Conference, Volume 7 Issue 1, October 2019, pp. page number

\begin{tabular}{cccc}
\hline Variable & $N$ & Mean & $S D$ \\
\hline Diet Practices & 66 & 15.58 & 3.81 \\
PhysicalActivity & 66 & 3.61 & 2.83 \\
\hline
\end{tabular}

Awareness on the factors causing non-communicable diseases is the first step to promote healthy diet and to acquire healthy practices. Knowledge about healthy diet are better learned in the universities and colleges. This result was supported by ASCD, Centers for Disease Control and Prevention (CDC)

in 2014, that formal and structured health education provides the knowledge and the skills that are required by students to make quality health decisions and thus enabling them to adopt healthy behaviors. This is expected to have been provided by their qualified teachers.

The Relationship of Knowledge on Healthy Lifestyle to Dietary Practices and Physical Activities

Table 2 presents the relationship of knowledge to dietary practices and physical activities of the university students in both the undergraduate and graduate schools, all staying in the dormitories. A correlation test via Pearson correlation was run and the data showed that there was no significant relationship between Knowledge level and Healthy Lifestyle (in terms of diet) level, and Knowledge level and Physical Activity level.

Table 2. Relationship of knowledge on healthy lifestyle to dietary practices and physical activities of the respondents.

\begin{tabular}{ccc}
\hline Components & Healthy Lifestyle (Diet) & Physical Activity \\
\hline Pearson Correlation & 0.051 & 0.033 \\
Sig. (2-tailed) & 0.687 & 0.795 \\
N & 66 & 66 \\
Interpretation & $\mathrm{NS}$ & $\mathrm{NS}$ \\
\hline
\end{tabular}

The result of this study is supported by a research conducted among medical and non-medical college students that suggests a superior knowledge about healthy lifestyle does not guarantee an adequate translation into practice with the barrier such as poor time management as seen to be the topmost reason for not being able to practice healthy dietary habits and exercise 
(Sajwani, et. al, 2009). Further, even medical and paramedical college students were wellinformed about the importance of healthy lifestyle and the possible consequences in nonadherence, yet they were not practicing it (Alissa, et al., 2015).

Physical activity is defined as any activity that uses energy which may bring about an increase in the depth of breathing and increased heart rate (USDA choosemyplate). It is known to offer numerous benefits such as reducing obesity among students (Wareham, Sluijs, \& Ekelund, 2005), better mental health (Smith, \& Biddle, 2008), and well-being (Ussher, Owen, \& Cook, 2007), however, research showed physical activity to decline with age though magnitude is ambiguous (Dumith, Gigante, \& Domingues, 2011). The decrease in physical activities among adolescent to early adulthood may be affected by change of environment, academic pressures, or extracurricular activities.

The Relationship of Knowledge of healthy lifestyle to Dietary Practices and Physical Activities when classified according to Age.

This presents the relationship of knowledge of healthy lifestyle to dietary practice and physical activities of the university students when classified according to Age.

Table 3 describes the result of the correlation test using Pearson correlation for respondents age 18-24 years old and Table 4 showed the result specific for respondents ages 24 and above.

Table 3. Relationship of knowledge level to Dietary practices and Physical Activity for respondents ages 18 to 24 years old.

\begin{tabular}{lcc}
\hline Components & Healthy Lifestyle (Diet) & Physical Activity \\
\hline Pearson Correlation & -0.164 & -0.168 \\
Sig. (2-tailed) & 0.338 & 0.328 \\
$\mathrm{~N}$ & 36 & 36 \\
Interpretation & $\mathrm{NS}$ & $\mathrm{NS}$ \\
\hline
\end{tabular}

Table 3 describes the result of the correlation test using Pearson correlation for respondents age 18-24 years old. The study showed no significant relationship among students ages 18 to 24 years old.

Similar research studies showed the difficulties that students living away from their families may have in the practice of healthy lifestyle, specifically on the taking a balanced diet and engaging in any form of physical exercise (Lupi, S., et al, 2015), thus results are not significant between the variables. It is important to highlight that lesser physical activities 

number

even for young students carries health risks, and may not exclude them from developing obesity that may have an ill-effect later in life. A research study showed that sedentary activities among young students were influenced by individual factors such as (lack of ) parental supervision, lack of self-discipline and seemed to be moderated by academic pressures and lack of time for engagement in physical exercise (Simons, D., 2014).

Table 4. Relationship of Knowledge Level to Dietary practices and Physical Activity for respondents ages 24 years old and above.

\begin{tabular}{ccc}
\hline Components & Healthy Lifestyle (Diet) & Physical Activity \\
\hline Pearson Correlation & $.387^{*}$ & $.463^{*}$ \\
Sig. (2-tailed) & 0.035 & 0.01 \\
$N$ & 30 & 30 \\
Interpretation & $\mathrm{S}$ & $\mathrm{S}$ \\
\hline
\end{tabular}

Table 4 presents the relationship of knowledge level to diet and physical activity for 24 years old and above. The study showed a significant relationship between the knowledge of healthy lifestyle to dietary practice and physical activity when grouped according to age; specifically only to those 24 years old and above with a 2-tailed result of (0.035) for Diet practices and result of (0.01) for Physical activity.

This table showed a significant relationship of knowledge to Diet and Physical activity only for 24 years and above which implied a not much engagement in physical activities among college students staying in dormitories. This is consistent with a study which showed that college students were likely to be sedentary, leading to unhealthy lives (Almutairi, et al., 2018), not mindful of their healthy diets and physical activities (Bagordo et al., 2013) due to attention to weights, the stress brought about by academic area and the beliefs (Driskell et al., 2005) making them feel inadequate example to others (Pearson \& Biddle, 2011), however, showed a better result in post graduate students. Students enrolled in health - related courses who practice healthy lifestyle behaviors are most likely to become confident in giving counsel to their patients about lifestyle change and most likely follows their advice (Bleich, et al, 2012). The significant result in healthy dietary practices and physical activity among 24 years and older may be related to their personal health responsibility as older adults. Study showed that health responsibility and control of stress had a better link with physical activity 
and proper nutrition which means, when an older adult college student have a higher health responsibility, the more evident is the practice of a healthy lifestyle (Al-Khawaldeh, 2014). Therefore, health promotion activities may be done at the student's level which can be done better by these older college students in their own dormitories as health mentors.

\section{DISCUSSION}

The aim of the study is to determine whether the knowledge on healthy lifestyle acquired by the university students has a relationship to its practice. This study pointed out that college students who are expected to have acquired knowledge on healthy lifestyle, which may have been part of their curriculum, does not guarantee a better practice in the daily lives of college students. This may be related to some factors which were not included in the study. The respondents of this study may have shown to be highly knowledgeable about healthy lifestyle however have fair results on dietary practices and moderate result on physical activities. A study showed that even if students are aware of the benefits of physical exercise, there was still a little engagement on any forms of physical activity among them, and in turn may have not taken enough amount of water (Kant \& Graubard, 2010). Another study resulted to dormitory life may seem to be another source of stress among medical students knowing the distance from their homes and the lack of family support (Baum \& Davis, 1980). Similar studies in Iran and India, with the use of gadgets and laptop usage as the cause of inactivity among students (Rani \& Sathiyasekaran, 2013).

Surprisingly, older adult students aged 24 years and above, showed to have a better application of the knowledge as shown in the study having significant results. Older adult students in the university may have acquired over time the so-called "personal health responsibility" which may have evidently shown a significant practice of a healthy lifestyle. The result may be in contrast to the previous study which reported a better score in the practice of healthy lifestyle among freshmen than the other levels of students as there is not much workload yet that are given to them (Alpar, Şenturan, Karabacak \& Sabuncu. 2008).

The present study was designed to assess the relationship of knowledge of healthy lifestyle to the practice of university students, grouping them according to age. The study found out that better practice of the healthy lifestyle are seen among older students. However, the result of the study may have limited to the level of practice only and did not include the range of activities that may have contributed to the cause of inactivity. 

number

As a result of the study, older university students may be proposed as the health mentors of the younger college students in the dormitories who can impart health promotion activities. Health promotion may be better at students' level with an easy accesibility of any exercise materials or equipment in their own dormitories

\section{Conclusion}

The knowledge of healthy lifestyle may not have a positive result if not being put into practice. It may just be a bunch of information that the students are hoarding throughout their years of stay in the university and not much of an aid in their daily living, if not applied. The study results revealed that respondents were highly knowledgeable on healthy lifestyle, have fair result on dietary practices and moderate result on the practice of physical activities. However, the significant result has been moderated by the age specifically among 24 years and above, who have enrolled in graduate studies. The study showed a gap in the physical activities the college students might be engaged in. This may be the time to start to engage themselves in some physical activities that increases their respiratory rates, thereby increasing their cardiac activities, as habits started during this stage may persist until adult life. To narrow the gap, it may be recommended that health promotion may be done at the student's level in their own dormitories specifically for older university students to act as health mentors of college students, to increase the accessibility to the use of the gym and reinforce the values of exercise and physical activity that will have immediate and long term health benefits for college students. Future research should include the development of instruments to detect a more extensive range of sedentary activities typical of college students.

\section{REFERENCES}

Alissa, E. M., H. Alsawadi, A. Zedan, D. Alqarni, M. Bakry and N. B. Hli. (2015). Knowledge, Attitude and Practice of Dietary and Lifestyle Habits Among Medical Students in King Abdulaziz University, Saudi Arabia. International Journal of Nutrition and Food Sciences, 4(6), 650-655.

Alpar SE, L. Şenturan, Ü. Karabacak, and N. Sabuncu. (2008). Change in the health promoting lifestyle behaviour of Turkish University nursing students from beginning to end of nurse training," Nurse Education in Practice, 8(6), 382-388,.

Al-Khawaldeh OA. (2014). Health promoting lifestyles of Jordanian university students. Int J Adv Nurs Stud, 3, 27-31. 
Almutairi, K. M., W. B. Alonazi, J. M. Vinluan, T. H. Almigbal, M. A. Batais, A. A. Alodhayani, R. I. Alhoqail. (2018). Health promoting lifestyle of university students in Saudi Arabia: a cross-sectional assessment. BMC public health, 18(1), 1093.

Al-Tamimi, T. R., H. A. Ba-Omar, and S. Nadar. (2017). Knowledge Regarding Secondary Prevention Lifestyle Practices Among Patients with Ischaemic Heart Disease in Oman: Pilot study. Sultan Qaboos University medical journal, 17(1), e88-e92.

ASCD, Centers for Disease Control and Prevention (CDC). (2014). Whole School, Whole Community, Whole Child: A Collaborative Approach to Learning and Health. Alexandria, VA: ASCD; 2014. Available at: http://www.ascd.ord/ASCD/pdf/siteASCD/publications/wholechild/wscc-acollaborative-approach.pdf. Accessed February 6, 2015.

Bagordo F, T . Grassi, F. Serio, A. Idolo and A. De Donno. (2013). Dietary habits and health among university students living at or away from home in southern Italy. Jo Food Nutr Res, 52(3),164-71.

Bano R, E AlShammari, SB Fatima, NA Al Shammari. (2013). A comparative study of Knowledge, Attitude, Practice of nutrition and non-nutrition student towards a balanced diet in Hail University. IOSR Journal of Nursing and Health Science, 2(3), 29-36. 650-655.

Basch C. (2011). Healthier children are better learners: A missing link in school reforms to close the achievement gap. 2010. http://www.equitycampaign.org/i/a/document/12557_EquityMattersVol6_Web03082 010.pdf.

Baum A, GE Davis. (1980). Reducing the stress of highdensity living: an architectural intervention. J Pers Soc Psychol, Mar, 38(3), 471-81.

Bleich S, WL. Bennett, KA. Gudzune and LA. Cooper. (2012). Impact of physician BMI on obesity care and beliefs. Obesity, 20(5), 999-1005.

Cotillard, A., S.P. Kennedy, L.C. Kong, E.Prifti, N. Pons, E. Le Chatelier, M. Almeida, B. Quinquis, F. Levenez, and N. Galleron, , et al, ANR. (2013). MicroObes consortium Dietary intervention impact on gut microbial gene richness Nature, 500, pp. 585

Driskell JA, YN. Kim and KJ. Goebel. (2005). Few differences found in the typical eating and physical activity habits of lower level and upper-level university students. $J$ Am Diet Assoc, 105(5), 798-801.

Dumith SC, DP. Gigante and M.R. Domingues et al. (2011). Physical activity change during adolescence: a systematic review and a pooled analysis. Int J Epidemiol, 40, 685-98.

Fotheringham MJ, R.L. Wonnacott and N. Owen. (2000). Computer use and physical inactivity in young adults: public health perils and potentials of new information technologies. Ann Behav Med, 22, 269-275. 
Frank E, E.H. Wright, M.K. Serdula, L.K. Elon and G. Baldwin. (2002). Personal and professional nutrition-related practices of US female physicians. Am J Clin Nutr, 75(2), 326-332.

Fontana, L. \& L. Partridge. (2015). Promoting health and longevity through diet: from model organisms to humans Cell, 161, 106-118

Howe M, A. Leidel, S.M. Krishnan, A. Weber, M. Rubenfire and E.A.Jackson. (2010). Patient-related diet and exercise counseling: do providers' own lifestyle habits matter? Prev Cardiol, 13(4), 180-185.

Hung OY, N.L. Keenan \& J. Fang. (2013). Physicians' health habits are associated with lifestyle counseling for hypertensive patients. Am J Hypertens, 26(2), 201-208.

Kant AK, Graubard BI. (2010). Contributors of water intake in US children and adolescents: associations with dietary and meal characteristics-National Health and Nutrition Examination Survey 2005-2006. Am J Clin Nutr, 92(4), 887-96.

Key TJ, N.E. Allen, E.A. Spencer \& R.C. Travis. (2002). The effect of diet on risk of cancer. LOancet, Sep 14, 360(9336), 861-8.

Lee R. E., \& A. C. King. (2003). Discretionary time among older adults: how do physical activity promotion interventions affect sedentary and active behaviors? Ann Behav Med, 25, 112-119.

Lupi, S., F. Bagordo, A. Stefanati, T. Grassi, L. Piccinni, M. Bergamini \& A. De Donno. (2015). Assessment of lifestyle and eating habits among undergraduate students in northern Italy. Ann Ist Super Sanità 2015, 51(2).

Pearson N., \& S. J. Biddle. (2011). Sedentary behavior and dietary intake in children, adolescents, and adults. A systematic review. Am J Prev Med, 41(2), 178-188.

Pengpid, S., K. Peltzer, H.K. Kassean, et al. (2015). Int J Public Health, 60, 539.

Rani A. M., \& B. W. Sathiyasekaran. (2013). Behavioural determinants for obesity: A crosssectional study among urban adolescents in India. J Prev Med Public Health, 46, 192200.

Sajwani, A. Rubina \& Shoukat, Sana \& Raza, Rushna \& S. Muhyeuddin, Muhannad \& Rashid, Quratulain \& S. Siddique, Momin \& Panju, Sukaina \& Raza, Hasan \& Chaudhry, Sophia \& Kadir and Muhannad. (2009). Knowledge and practice of healthy lifestyle and dietary habits in medical and non-medical students of Karachi, Pakistan. JPMA. The Journal of the Pakistan Medical Association, 59, 650-5.

Simons D, P. Clarys, I. De Bourdeaudhuij, B. de Geus, C. Vandelanotte, B. Deforche. (2014). Why do Young Adults Choose Different Transport Modes? Transport Policy: A focus group study. 
Sonnenburg, E. D., S. A. Smits., M. Tikhonov, S. K Higginbottom, N. S. Wingreen and J.L. Sonnenburg (2016). Diet-induced extinctions in the gut microbiota compound over generations Nature, 529, 212-215

Smith A, S. Biddle, eds. (2008). Youth physical activity and sedentary behavior. Champaign, IL: HumanKinetics

Strong WB, R.M. Malina, C.J. Blimkie, S.R. Daniels, R.K. Dishman, B.Gutin, A.C. Hergenroeder, A. Must,P.A. Nixon, J.M. Pivarnik, T. Rowland, S.Trost and F. Trudeau. (2005) c. Evidence based physical activity for school-age youth. Journal of Pediatrics, 146(6), 732-737.

Ussher MH, CG Owen and DG Cook, et al. (2007). The relationship between physical activity, sedentary behaviour and psychological wellbeing among adolescents. Soc Psychiatry Psychiatr Epidemiol, 42, 851-6.

Utter J, Scragg R, Mhurchu CN, Schaaf D. (2007). At-home breakfast consumption among New Zealand children: Associations with body mass index and related nutrition behaviors. J Am Diet Assoc, 107, 570-6.

U.S. Department of Agriculture and U.S. Department of Health and Human Services. (2015). Dietary Guidelines for Americans. 8th ed. Washington, DC: US Government Printing Office.

Wareham NJ, EM van Sluijs and U. Ekelund. (2005). Physical activity and obesity prevention: a review of the current evidence. Proc Nutr Soc, 64, 229-47.

Zakaria, A. \& Z. Syahirah, Z. Abidin. (2014). Knowledge and Practice of Healthy Lifestyle Among Higher Institution Student. Proceedings of International Academic Conferences 0100729, International Institute of Social and Economic Sciences. 